\title{
The relationship between intimate partner violence and HIV outcomes among pregnant women living with HIV in Malawi
}

Elizabeth C. Wetzel ${ }^{1,2}$, Tapiwa Tembo ${ }^{2}$, Elaine J. Abrams ${ }^{4}$, Alick Mazenga ${ }^{2}$, Mike J. Chitani ${ }^{2}$, Saeed Ahmed ${ }^{1,2}$, Xiaoying Yü ${ }^{3}$, Maria H. Kim ${ }^{1,2}$

\author{
1. Baylor College of Medicine International Pediatric AIDS Initiative, Texas Children's Hospital, Houston, United States of America (USA) \\ 2. Baylor College of Medicine - Children's Foundation Malawi, Lilongwe, Malawi \\ 3. University of Texas Medical Branch at Galveston, Galveston, USA \\ 4. ICAP at Columbia University, Mailman School of Public Health and Department of Pediatrics, Vagelos College of Physicians \& Surgeons, \\ Columbia University, New York, USA
}

*Corresponding Author: Elizabeth C. Wetzel; Email: ecwetzel@bcm.edu

\section{Background}

\section{Abstract}

Intimate partner violence (IPV) is a global public health concern particularly in pregnancy where IPV can have negative health implications for the mother and child. Data suggest IPV disproportionately affects pregnant women living with HIV (PWLWH) compared to those without HIV. HIV-related outcomes are worse among women experiencing IPV. Despite this knowledge, there is paucity of data concerning PWLWH and IPV in Malawi, where there is a high HIV prevalence (10.6\%).

Objectives

We aim to characterize IPV amongst PWLWH in Malawi and describe its relationship to demographic characteristics, psychosocial factors, and HIV-related outcomes.

Methods

This analysis used data from the VITAL Start pilot study, which is a video-based intervention targeting retention and ART adherence amongst PWLWH in Malawi. PWLWH not on ART were recruited at antenatal clinic and given study questionnaires to assess demographics, IPV, and psychosocial factors. Questionnaires were also administered at one-month follow-up to assess outcomes related to HIV. Descriptive statistics and logistic regression models were used to explore the risk factors associated with IPV.

\section{Results}

Thirty-nine percent of participants reported ever experiencing IPV from their current partner. The majority (53\%) reporting IPV experienced more than one type of violence. IPV was associated with being married $(\mathrm{p}=0.04)$ and depression $(\mathrm{p}<0.0001)$ in the univariable analysis. For women retained at one-month, IPV was associated with reporting a missed ART dose in the past month but not with adherence measured by pill count.

\section{Conclusions}

A large proportion of PWLWH experienced IPV from their current partner and IPV was associated with worse self-reported ART adherence at one-month follow-up. Further evidence is needed to understand how IPV impacts PWLWH throughout postpartum and beyond. Given the detrimental impact on health outcomes among PWLWH in Malawi, additional focus on IPV is essential to identify mechanisms to prevent, screen, and manage IPV among this population.

Key words: IPV, abuse, HIV, Malawi, adherence, violence

\section{Introduction}

Globally, intimate partner violence (IPV) is a public health concern, including in the sub-Saharan African region where current prevalence estimates $44 \%$ of women have experienced IPV. ${ }^{1}$ IPV includes any behavior within an intimate relationship that causes physical, psychological, or sexual harm to those in the relationship. ${ }^{2}$ Intimate partner violence can negatively affect the physical and emotional well-being of the victim and can have downstream health consequences. In the context of pregnancy, IPV can detrimentally impact both maternal and neonatal health outcomes. ${ }^{3-10}$

Data suggest IPV rates among pregnant women may be higher amongst pregnant women living with HIV (PWLWH) compared to non-infected pregnant women. ${ }^{11,12}$ In subSaharan Africa estimates of IPV among PWLWH range from $20 \%-60 \% \cdot{ }^{13-16}$ In one South African study, PWLWH without a history of IPV had a higher risk of IPV after pregnancy, compared to non-infected pregnant women. ${ }^{17}$

IPV has been linked to worse antiretroviral treatment (ART) adherence and viral load suppression in women living with HIV (WLWH). ${ }^{18-20}$ PWLWH already face substantial challenges with ART adherence and retention in care ${ }^{21}$ and IPV during pregnancy can compound these challenges. ${ }^{20}$ Furthermore, IPV during pregnancy can also negatively impact mental health outcomes and has been associated with a higher risk of depression. ${ }^{22-24}$ Experiencing IPV during pregnancy may increase the odds of postpartum depression, ${ }^{25}$ and depression has been linked to worse HIV outcomes such as ART non-adherence, virologic failure, and AIDS-related mortality. ${ }^{26-28}$

In Malawi, the lifetime prevalence of IPV is estimated to be $38 \%$ among ever-partnered women aged $15-49 .{ }^{29}$ However, 
rates of IPV during pregnancy and among PWLWH have not been well documented. Estimates of IPV during pregnancy among Malawian women vary widely, from 5\% from the Malawi Demographic Health Survey in $2015,{ }^{30}$ to $21 \%$ in a reproductive health study in Lilongwe District, ${ }^{8}$ and up to $59 \%$ from a recent study conducted in Nsanje District. ${ }^{31}$ Despite evidence from other countries in the African region that risk of IPV is higher amongst PWLWH, and that IPV detrimentally impacts HIV treatment and health outcomes, no published data on IPV exists among PWLWH in Malawi.

This analysis aims to address this gap by 1) characterizing IPV amongst PWLWH in Lilongwe, Salima, and Mangochi Districts in Malawi and 2) describing its relationship to demographic and psychosocial factors, as well as its relationship to partner disclosure, ART adherence, and retention in ART clinic.

\section{Methods}

\section{Setting}

This analysis used data from a study that examined the acceptability, feasibility, and early implementation outcomes of a video-based intervention on retention and ART adherence amongst pregnant women living with HIV in Malawi (VITAL Start, Video-intervention to inspire treatment adherence for life). ${ }^{32}$ The study took place in three government health facilities in central and southern Malawi which offer free HIV and ART services. The study sites are an urban health facility in Lilongwe and semi-urban health facilities in Mangochi and Salima districts. In Malawi, over 95\% of pregnant women between 2010-2015 attended at least one antenatal care (ANC) visit and $>90 \%$ give birth at a health facility. ${ }^{30} \mathrm{HIV}$ testing coverage at ANC is $>90 \%$ for the first clinic visit as per Malawi Ministry of Health $(\mathrm{MOH})$ HIV testing guidance. ${ }^{30}$ Prevalence of $\mathrm{HIV}$ in Malawi is $10.6 \%$ among the adult population, and higher among women $(12.8 \%) .^{33}$

All pregnant women identified in ANC as HIV positive and not on ART were offered pre-ART counselling (randomized to either VITAL Start or standard of care). Both VITAL Start and standard of care included counselling on the importance of adhering to ART and encouraged partner disclosure. Firstline ART regimen for all women newly initiating treatment at the time of this study was a fixed-dose combination pill containing tenofovir, lamivudine, and efavirenz.

\section{Study Population Eligibility and Recruitment}

Detailed information regarding VITAL Start has been published. ${ }^{32}$ In brief, PWLWH not receiving ART who presented for antenatal care between December 2016 to Feb 2018 were eligible for recruitment. Other eligibility criterion included age $\geq 18$ years, or if she was married or had a previous child then she could be $\geq 16$ years; understands Chichewa; no disabilities that would prevent her from viewing or understanding the intervention video; residence in the health center catchment area for 12 months. Women identified as eligible were informed about the study by trained research assistants (RA). Baseline questionnaires were administered at enrollment and a follow-up questionnaire was given at onemonth post enrollment assessing self-reported adherence and behavioral factors that affect women's ability to start and remain on an ART regimen. Intimate partner violence (IPV) was assessed at enrollment. One-month follow up surveys were conducted at the clinic. If the participant did not return for the survey, she was traced using locator information (up to three phone call attempts and home visit attempts).

\section{Data collection}

Trained RAs administered baseline and one-month questionnaires that were translated into Chichewa and backtranslated to English. Items were pretested with potential participants to validate the language in the culture and context. Questionnaires were administered face-to-face and study follow-up visits were scheduled separately from clinic visits to avoid biasing the main outcome. Surveys were collected on paper and responses were entered in an Excel spreadsheet embedded with validations to ensure data quality. HIV related clinical data were extracted from patient medical records. Data were reviewed for accuracy and completeness.

\section{Measures}

\section{Sociodemographics}

Sociodemographic survey items included age, literacy, education, income, employment, marital status, pregnancy intention, condom use, number of sexual partners in the last year, cohabitation with partner, partner's age, and time since HIV diagnosis. For marital status we asked the participant if she was married or had a partner (which was classified as "steady partner"). Women without a partner were not asked IPV questions and therefore not included in the analysis. Partner's HIV status was reported by the participant.

\section{Intimate Partner Violence and Other Psychosocial Outcomes (Depression, social support, alcohol and drug use)}

We assessed psychosocial and behavioral characteristics using locally validated scales. IPV was measured using the World Health Organization (WHO) intimate partner violence survey. ${ }^{34,35}$ IPV was only assessed on women who reported having a partner (either married or steady partner) and collected only at baseline (at enrollment). IPV had binary responses (Yes/No) for a total of 13 items and assessed ever having experienced sexual (e.g., "Has he physically forced you to have sexual intercourse when you did not want to?"), emotional (e.g., "Has he insulted you or made you feel bad about yourself?"), or physical violence (e.g. "Has he slapped you or thrown something at you that could hurt you?") from the participant's current partner. Any 'Yes' response was classified as experiencing IPV from the current partner. Additional psychosocial measurements included depression WHO self-reporting questionnaire (SRQ), 36,37 social support (multi-dimensional scale of perceived social support (MSPSS), ${ }^{38,39}$ alcohol use (alcohol use disorders identification test (AUDIT)), ${ }^{40-42}$ and drug use (drug use disorders identification test (DUDIT). ${ }^{43-45}$

WHO SRQ included a 20-item survey with binary responses (Yes/No). Depression was categorized into low, medium, and high using cutoffs of 4 and $8 .{ }^{37}$ MSPSS included 12 items scored on a 5 -point scale, with a possible total score ranging from 5-60. MSPSS was sub-categorized into three groups including other, family, and friends, with possible score ranging from 5-20. A higher score indicates a higher level of the participants' perceived social support. ${ }^{23}$ When calculating the summary scale, 'Refuse to answer' responses were considered missing. We used means calculated from the available items from the same scale and same individual. For SRQ, we imputed from individuals that completed at least $80 \%$ of items for SRQ. The imputed results were reported. The AUDIT had 10 items with a maximum score of 40 . 
Table 1. Descriptive Statistics of study population, $N=262$

\begin{tabular}{|c|c|c|c|c|}
\hline & No IPV & Any IPV & Total & \\
\hline Variable & $\mathrm{n}(\%$ or $\mathrm{SD})$ & $\mathrm{n}(\%$ or $\mathrm{SD})$ & $\mathrm{n}(\%$ or $\mathrm{SD})$ & $p$-value \\
\hline Total included in study population & $161(61.5)$ & $101(38.5)$ & 262 & \\
\hline Age, years, mean (SD) & $27.0(5.4)$ & $27.6(5.8)$ & $27.2(5.5)$ & 0.38 \\
\hline \multicolumn{5}{|l|}{ Education, $\mathrm{n}(\%)$} \\
\hline None & $17(70.8)$ & $7(29.2)$ & $24(9.2)$ & \multirow{6}{*}{0.48} \\
\hline Some primary (standard 1-5) & $40(58.0)$ & $29(42.0)$ & $69(26.3)$ & \\
\hline Primary (Standard 6-8) & $44(57.1)$ & $33(42.9)$ & $77(29.4)$ & \\
\hline Junior High School & $32(59.3)$ & $22(40.7)$ & $54(20.6)$ & \\
\hline High School/ Secondary school & $24(75.0)$ & $8(25.0)$ & $32(12.2)$ & \\
\hline Post-secondary & $4(66.7)$ & $2(33.3)$ & $6(2.3)$ & \\
\hline \multicolumn{5}{|l|}{ Literate, n (\%) } \\
\hline No & $41(60.3)$ & $27(39.7)$ & $68(26)$ & \multirow{3}{*}{0.95} \\
\hline Yes & $118(61.8)$ & $73(38.2)$ & $191(72.9)$ & \\
\hline Missing & $2(66.7)$ & $1(33.3)$ & $3(1.1)$ & \\
\hline \multicolumn{5}{|l|}{ Monthly Income, n (\%) } \\
\hline Less than $49,999 \mathrm{MK}$ & $109(64.1)$ & $61(35.9)$ & $170(64.9)$ & \multirow{6}{*}{0.63} \\
\hline Between 50,000 and $99,999 \mathrm{MK}$ & $26(52.0)$ & $24(48.0)$ & $50(19.1)$ & \\
\hline Between 100,000 and $249,999 \mathrm{MK}$ & $16(64.0)$ & $9(36.0)$ & $25(9.5)$ & \\
\hline $250,000 \mathrm{MK}$ or more & $5(62.5)$ & $3(37.5)$ & $8(3.1)$ & \\
\hline Missing & $1(100.0)$ & $0(0.0)$ & $1(0.4)$ & \\
\hline Refuse to answer & $4(50.0)$ & $4(50.0)$ & $8(3.1)$ & \\
\hline \multicolumn{5}{|l|}{ Intended to become pregnant, $\mathrm{n}(\%)$} \\
\hline No & $30(58.8)$ & $21(41.2)$ & $51(19.5)$ & \multirow{3}{*}{0.41} \\
\hline Yes & $131(62.4)$ & $79(37.6)$ & $210(80.2)$ & \\
\hline Refuse to answer & $0(0.0)$ & $1(100.0)$ & $1(0.4)$ & \\
\hline \multicolumn{5}{|l|}{ Condom use before pregnancy, $n(\%)$} \\
\hline No & $137(61.4)$ & $86(38.6)$ & $223(85.1)$ & \multirow{4}{*}{0.23} \\
\hline Yes, sometimes & $21(70.0)$ & $9(30.0)$ & $30(11.5)$ & \\
\hline Yes, most times & $2(40.0)$ & $3(60.0)$ & $5(1.9)$ & \\
\hline Yes, all the time & $1(25.0)$ & $3(75.0)$ & $4(1.5)$ & \\
\hline \multicolumn{5}{|l|}{ Time of HIV diagnosis, $n(\%)$} \\
\hline Today & $153(61.4)$ & $96(38.6)$ & $249(95)$ & \multirow{3}{*}{1.0} \\
\hline Before today, but during this pregnancy & $5(62.5)$ & $3(37.5)$ & $8(3.1)$ & \\
\hline Before today, not during this pregnancy & $3(60.0)$ & $2(40.0)$ & $5(1.9)$ & \\
\hline \multicolumn{5}{|l|}{ Number of sexual partners in last year, $n(\%)$} \\
\hline 1 & $136(60.7)$ & $88(39.3)$ & $224(85.5)$ & \multirow{3}{*}{0.25} \\
\hline 2 or more & $20(60.6)$ & $13(39.4)$ & $33(12.6)$ & \\
\hline Missing & $5(100.0)$ & $0(0.0)$ & $5(1.9)$ & \\
\hline \multicolumn{5}{|l|}{ Marital Status, n (\%) } \\
\hline Married & $143(59.6)$ & $97(40.4)$ & $240(91.6)$ & \multirow[t]{2}{*}{0.04} \\
\hline Steady partner & $18(81.8)$ & $4(18.2)$ & $22(8.4)$ & \\
\hline \multicolumn{4}{|l|}{ Currently living with partner, $\mathrm{n}(\%)$} & \multirow{3}{*}{0.7} \\
\hline No & $21(65.6)$ & $11(34.4)$ & $32(12.2)$ & \\
\hline Yes & $140(60.9)$ & $90(39.1)$ & $230(87.8)$ & \\
\hline
\end{tabular}


Table 1 Cont..

\begin{tabular}{|c|c|c|c|c|}
\hline Partner's approximate age, $\mathrm{n}(\%)$ & $\begin{array}{c}\text { No IPV } \\
\mathrm{n}(\% \text { or SD) }\end{array}$ & $\begin{array}{c}\text { Any IPV } \\
\mathrm{n}(\% \text { or SD) }\end{array}$ & $\begin{array}{c}\text { Total } \\
\mathrm{n}(\% \text { or SD) }\end{array}$ & \multirow{5}{*}{0.96} \\
\hline 24 years or younger & $15(65.2)$ & $8(34.8)$ & $23(8.8)$ & \\
\hline $25-34$ years & $82(60.3)$ & $54(39.7)$ & $136(51.9)$ & \\
\hline 35 years or older & $63(62.4)$ & $38(37.6)$ & $101(38.5)$ & \\
\hline Refuse to answer & $1(50.0)$ & $1(50.0)$ & $2(0.8)$ & \\
\hline \multicolumn{5}{|l|}{ Partner's current HIV status, n (\%) } \\
\hline Negative & $36(56.3)$ & $28(43.8)$ & $64(24.4)$ & \multirow{4}{*}{0.78} \\
\hline Positive & $14(63.6)$ & $8(36.4)$ & $22(8.4)$ & \\
\hline Never test/Inconclusive/Don't know & $110(62.9)$ & $65(37.1)$ & $175(66.8)$ & \\
\hline Refuse to answer & $1(100.0)$ & $0(0.0)$ & $1(0.4)$ & \\
\hline \multicolumn{5}{|l|}{$\mathrm{SRQ}^{\S}$ total score (mean imputation), $\mathrm{n}(\%)$} \\
\hline Low (0-4) & $123(72.8)$ & $46(27.2)$ & $169(64.5)$ & \multirow{4}{*}{$<0.0001$} \\
\hline Medium $(>4-<8)$ & $20(50.0)$ & $20(50.0)$ & $40(15.3)$ & \\
\hline High $(8+)$ & $18(34.6)$ & $34(65.4)$ & $52(19.8)$ & \\
\hline Missing & $0(0.0)$ & $1(100.0)$ & $1(0.4)$ & \\
\hline \multicolumn{5}{|l|}{ AUDIT* total score, $n(\%)$} \\
\hline Non-drinker $(0)$ & $145(62.0)$ & $89(38.0)$ & $234(89.3)$ & \multirow{4}{*}{0.90} \\
\hline Low risk (1-7) & $4(57.1)$ & $3(42.9)$ & $7(2.7)$ & \\
\hline High risk $(\geq 8)$ & $2(50.0)$ & $2(50.0)$ & $4(1.5)$ & \\
\hline Missing & $10(58.8)$ & $7(41.2)$ & $17(6.5)$ & \\
\hline \multicolumn{5}{|l|}{$\mathrm{DUDIT}^{ \pm}$total score, $\mathrm{n}(\%)$} \\
\hline No drug-related problem (0) & $147(61.5)$ & $92(38.5)$ & $239(91.2)$ & \multirow{3}{*}{1} \\
\hline Drug-related problem $(\geq 2)$ & $1(50.0)$ & $1(50.0)$ & $2(0.8)$ & \\
\hline Missing & $13(61.9)$ & $8(38.1)$ & $21(8)$ & \\
\hline $\begin{array}{l}\text { MSPSS } \beta \text { significant others total score (complete items), mean } \\
\text { (SD) }\end{array}$ & $15.7(1.8)$ & $15.6(2.0)$ & $15.7(1.9)$ & 0.51 \\
\hline MSPSS family total score (complete items), mean (SD) & $14.9(2.5)$ & $15.0(2.8)$ & $14.9(2.6)$ & 0.87 \\
\hline MSPSS friends total score (complete items), mean (SD) & $14.3(2.8)$ & $13.7(3.0)$ & $14.1(2.9)$ & 0.15 \\
\hline MSPSS total score (complete items), mean (SD) & $44.9(5.8)$ & $44.3(6.1)$ & $44.7(5.9)$ & 0.42 \\
\hline
\end{tabular}

§Self-Reporting Questionnaire, WHO

¥Alcohol use disorders identification test

${ }^{ \pm}$Drug use disorders identification test

\section{${ }^{\beta}$ Multi-dimensional scale of perceived social support}

Scores of zero were classified as non-drinkers, while 1-7 was classified as low-risk alcohol use and $\geq 8$ was classified as harmful alcohol use. ${ }^{46}$ The DUDIT had 11 items and a score of $\geq 2$ indicated a possible drug related problem. ${ }^{43}$ For both AUDIT and DUDIT, those with missing responses on the first question (which indicates whether she drinks or uses drugs at all) or reported as an alcohol/drug user but with missing responses on all following items were treated as missing data.

\section{HIV Outcomes Measures}

Surveys included HIV-related information such as adherence self-efficacy (ASE) (which assessed how well she expects to remain adherent to ART medication), ${ }^{47,48}$ partner disclosure information (if her partner knew her status, when disclosure occurred, and who disclosed her status), ${ }^{49}$ and self-reported adherence. $^{50,51}$ Date of ART start, pill count data, and one- month retention data were abstracted from patient medical records.

Adherence self-efficacy (ASE) was scored on a 4-point scale (1-4) with a total possible score of 44 signifying the highest level of perceived efficacy. Mean scores from the one-month follow up visit as well as the change score (month 1 minus baseline score) were calculated. For missing data, we reported imputed values from individuals that completed at least $80 \%$ of items for ASE.

Participants who had a recorded ART clinic visit 14-61 days after ART initiation were considered retained at one-month. Behavioral adherence was measured by self-report and pill count. For pill count the ratio of total number of pills given during the enrollment visit divided by the follow up time calculated the proportion of days covered (PDC). ${ }^{52}$ 
Table 2. Univariable logistic regression (outcome: any IPV experienced by current partner)

\begin{tabular}{|c|c|c|c|c|c|}
\hline Variable & $\begin{array}{c}\text { Mean }(S D, n) / \\
n(\%)\end{array}$ & IPV Yes n(\%) & OR $(95 \% \mathrm{Cl})$ & $p$-value & $\begin{array}{l}\text { p-value } \\
\text { (overall) }\end{array}$ \\
\hline $\begin{array}{l}\text { MSPSS friends total score (complete } \\
\text { items) }\end{array}$ & $14.1(2.9,260)$ & & $0.94[0.86-1.02]$ & 0.16 & 0.16 \\
\hline MSPSS total score (complete items) & $44.7(5.9,259)$ & & $0.98[0.94-1.03]$ & 0.42 & 0.42 \\
\hline \multicolumn{5}{|l|}{ Education } & \multirow{7}{*}{0.12} \\
\hline None & $24(9.2)$ & $7(29.2)$ & $1.15[0.37-3.60]$ & 0.81 & \\
\hline $\begin{array}{l}\text { Some primary/ Primary/ Middle } \\
\text { School (JCE) }\end{array}$ & $200(76.3)$ & $84(42.0)$ & $2.03[0.93-4.40]$ & 0.07 & \\
\hline $\begin{array}{l}\text { High school (MCSE) /Post- } \\
\text { secondary }\end{array}$ & $38(14.5)$ & $10(26.3)$ & Reference & . & \\
\hline \multicolumn{5}{|l|}{ Literate } & \\
\hline No & $68(26.3)$ & $27(39.7)$ & $1.06[0.60-1.88]$ & 0.83 & \\
\hline Yes & 191 (73.7) & $73(38.2)$ & Reference & . & \\
\hline \multicolumn{5}{|l|}{ Monthly income } & \multirow{6}{*}{0.25} \\
\hline Less than $49,999 \mathrm{MK}$ & $170(67.2)$ & $61(35.9)$ & $0.73[0.43-1.25]$ & 0.25 & \\
\hline$\geq 50,000 \mathrm{MK}$ & $83(32.8)$ & $36(43.4)$ & Reference & . & \\
\hline Pregnancy intention & & & & & \\
\hline No & $51(19.5)$ & $21(41.2)$ & $1.16[0.62-2.17]$ & 0.64 & \\
\hline Yes & $210(80.5)$ & $79(37.6)$ & Reference & . & \\
\hline \multicolumn{5}{|l|}{ Time of HIV diagnosis } & \multirow{6}{*}{0.0495} \\
\hline Today & $249(95.5)$ & $96(38.6)$ & $1.0[0.32-3.16]$ & 0.99 & \\
\hline Before today & $13(5.5)$ & $5(38.5)$ & Reference & . & \\
\hline Marital status & & & & & \\
\hline Married & 240 (91.6) & $97(40.4)$ & $3.05[1.00-9.30]$ & 0.0495 & \\
\hline Steady partner & $22(8.4)$ & $4(18.2)$ & Reference & . & \\
\hline \multicolumn{5}{|l|}{ Currently living with partner } & \multirow{7}{*}{$<.0001$} \\
\hline No & $32(12.2)$ & $11(34.4)$ & $0.81[0.37-1.77]$ & 0.61 & \\
\hline Yes & $230(87.8)$ & $90(39.1)$ & Reference & . & \\
\hline SRQ total score (mean imputation) & & & & & \\
\hline Low $(0-4)$ & $169(64.8)$ & $46(27.2)$ & 0.20 [0.10-0.38] & $<.0001$ & \\
\hline Medium $(>4-<8)$ & $40(15.3)$ & $20(50.0)$ & $0.53[0.23-1.23]$ & 0.14 & \\
\hline High (8+) & $52(19.9)$ & $34(65.4)$ & Reference & . & \\
\hline \multicolumn{5}{|l|}{ AUDIT total score } & \multirow{3}{*}{0.62} \\
\hline Non-drinker & $234(95.5)$ & $89(38.0)$ & $0.74[0.22-2.48]$ & 0.62 & \\
\hline Low/high risk & $11(4.5)$ & $5(45.5)$ & Reference & . & \\
\hline
\end{tabular}

The follow-up time was calculated as the duration between ART start date and the one-month visit during the 14-61 day retention period. If the PDC exceeded 1 , the value was capped at 1 . Adherence was categorized using a cutoff of 0.9. Participants with at least $90 \%$ pills covered in the interval were considered adherent, and those with $<90 \%$ pills covered were considered not adherent. ${ }^{53}$ Among those retained at one month, participants with missing pill count data prior to the visit were considered as a separate category (pills not given). To measure self-reported adherence, participants were asked at the monthly follow-up visit if they had missed any doses in the past 7 and 30 days.

\section{Data Analysis}

Descriptive statistics, such as mean and standard deviation (SD) for continuous variables, and frequency and proportion for categorical variables, were calculated among all subjects and by IPV status. Chi-Squared test/Fisher's exact test was used to compare the distribution of the categorical variables (proportions) between two groups. Both tests yielded consistent results and p-values from Fisher's exact tests were reported. For the continuous variables, two sample t-test was used to compare the means between two groups.

Logistic regression models were used to explore the risk factors associated with any IPV. Variables of interest or 
Table 3. HIV Outcomes at month one by IPV experienced by current partner

\begin{tabular}{|c|c|c|c|c|}
\hline Variable & No IPV & Yes IPV & Total & $p$-value \\
\hline \multicolumn{4}{|l|}{ Started ART, n (\%) } & \multirow{4}{*}{0.92} \\
\hline No & $1(0.6)$ & $0(0.0)$ & $1(0.4)$ & \\
\hline Yes & $134(83.2)$ & $86(85.1)$ & $220(84.0)$ & \\
\hline Missing & $26(16.1)$ & $15(14.9)$ & $41(15.6)$ & \\
\hline \multicolumn{4}{|l|}{ Disclosed to Partner, n (\%) } & \multirow{6}{*}{0.48} \\
\hline No & $16(9.9)$ & $12(11.9)$ & $28(10.7)$ & \\
\hline Yes & $92(57.1)$ & $62(61.4)$ & $154(58.8)$ & \\
\hline Already disclosed & $29(18)$ & $12(11.9)$ & $41(15.6)$ & \\
\hline Missing & $24(14.9)$ & $14(13.9)$ & $38(14.5)$ & \\
\hline Refuse to answer & $0(0.0)$ & $1(1.0)$ & $1(0.4)$ & \\
\hline \multicolumn{4}{|l|}{ Retained in ART clinic, n (\%) } & \multirow{3}{*}{0.55} \\
\hline No & $39(24.2)$ & $21(20.8)$ & $60(22.9)$ & \\
\hline Yes & $122(75.8)$ & $80(79.2)$ & $202(77.1)$ & \\
\hline \multicolumn{4}{|l|}{ Adherence (Pill count), n (\%) } & \multirow{5}{*}{0.71} \\
\hline$<90 \%$ & $21(13.0)$ & $15(14.9)$ & $36(13.7)$ & \\
\hline$\geq 90 \%$ & $100(62.1)$ & $63(62.4)$ & $163(62.2)$ & \\
\hline Missing & $39(24.2)$ & $21(20.8)$ & $60(22.9)$ & \\
\hline Pill not given & $1(0.6)$ & $2(2.0)$ & $3(1.1)$ & \\
\hline \multicolumn{4}{|l|}{ Missed dose in past week, n (\%) } & \multirow{4}{*}{0.08} \\
\hline No & $112(69.6)$ & $60(59.4)$ & $172(65.6)$ & \\
\hline Yes & $21(13.0)$ & $24(23.8)$ & 45 (17.2) & \\
\hline Missing & $28(17.4)$ & $17(16.8)$ & $45(17.2)$ & \\
\hline \multicolumn{4}{|l|}{ Missed dose in past month, $\mathrm{n}(\%)$} & \multirow{5}{*}{0.0499} \\
\hline No & $107(66.5)$ & $56(55.4)$ & $163(62.2)$ & \\
\hline Yes & $23(14.3)$ & $26(25.7)$ & $49(18.7)$ & \\
\hline Missing & 31 (19.3) & $18(17.8)$ & $49(18.7)$ & \\
\hline Refuse to answer & $0(0.0)$ & $1(1.0)$ & $1(0.4)$ & \\
\hline $\begin{array}{l}\text { ASE, total score (mean imputation), } \\
\text { mean (SD) }\end{array}$ & $35.2(5.2)$ & $35.6(5.2)$ & $35.4(5.2)$ & 0.58 \\
\hline $\begin{array}{l}\text { ASE, Change in total score (mean } \\
\text { imputation), mean (SD) }\end{array}$ & $0.8(5.5)$ & $1.7(6.2)$ & $1.1(5.8)$ & 0.28 \\
\hline
\end{tabular}

with clinical importance were considered in the modeling. We first performed univariable logistic regression models for all variables and those with p-values $<0.2$ were included in the model selection in multivariable logistic model. Some response categories were combined due to sparse cells or similar outcomes. The scale of the continuous variables was examined using smoothing spline techniques and quartile method to ensure that the assumption of linearity on the logit was appropriate. A backwards selection procedure was performed to select variables and those achieving statistical significance were retained in the model. The overall model fit was examined using Hosmer-Lemeshow test. The odds ratio (OR) estimates and their 95\% confidence intervals (CI) were reported for the assessment on the strength of the associations.

All tests were two-sided. A p-value of 0.05 was deemed statistically significant. All analyses were performed using SAS software 9.4 (SAS Institute, Inc., North Carolina, USA).

\section{Ethical Considerations}

As per the larger study protocol, we obtained written, informed consent from all study participants. Participants who reported any IPV, suicidal ideation, high level of depression, drug/alcohol abuse, or any women exhibiting severe distress during the interview were referred to a trained, on-site psychosocial counsellor. The National Health Sciences Research Committee (NHSRC) of the Malawi Ministry of Health and the Baylor College of Medicine Institutional Review Board in the USA approved the study protocol.

\section{Results}

\section{Cohort Characteristics}

Of the 306 women enrolled in the VITAL Start pilot study, thirty-seven were excluded because they did not have partners so they were not asked IPV questions, and seven were excluded because they had missing data for IPV, therefore 262 women were eligible and and included in the analysis. Demographic and cohort characteristics can be found in Table 1. Mean (SD) age of study participants was 27 (5.5) years, 92\% were married $(n=240), 65 \%(n=169)$ completed primary school education (Standard 6-8), and 84\% reported a household monthly income of $<100,000$ Malawi Kwacha (roughly 140 USD) $(n=220)$. Most women learned their HIV diagnosis at the ANC visit during the time of recruitment $(95 \%, \mathrm{n}=249)$ and two-thirds $(67 \%, \mathrm{n}=175)$ reported not https://dx.doi.org/10.4314/mmj.v33i4.4 


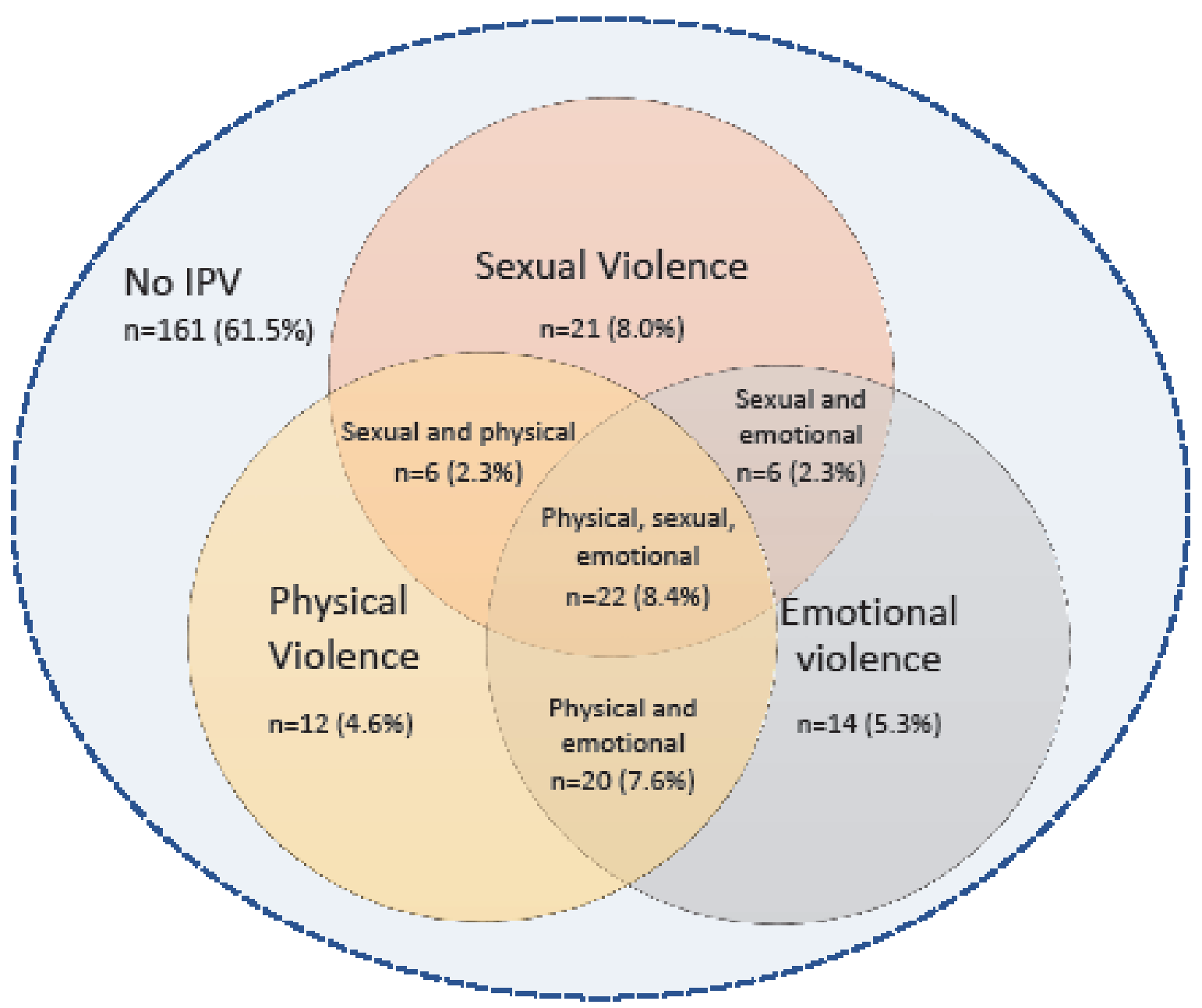

Figure 1. IPV experienced by current partner among study population $(n=262)$

knowing their partner's HIV status. Few women $(11 \%, n=28)$ reporting ever drinking alcohol, and only four $(1.5 \%)$ scored as a high-risk drinker. Similarly, only two women reported a drug-related problem $(<1 \%)$.

\section{IPV Prevalence and Factors associated with IPV}

Thirty-nine percent of participants reported experiencing at least one form of IPV from their current partner $(n=101)$. Mean age of PWLWH experiencing IPV was 27.6 years. All three forms of IPV occurred at similar frequency (emotional: $24 \%, \mathrm{n}=62$; physical: 23\%, $\mathrm{n}=60$; sexual: $21 \%, \mathrm{n}=55$ ) (Figure 1). Among participants experiencing IPV, many experienced more than one type of IPV $(53 \%, \mathrm{n}=54)$. Twenty-two $(8 \%)$ participants experienced all three measured forms of violence. In univariable analysis being married $(p=0.04)$ and depression $(p<0.0001)$ were the only characteristics associated with experiencing IPV. The univariable logistic regression model showed that with each unit increase in MSPSS friends total score, there was a $6 \%$ decrease in the odds of having any IPV, although this was not statistically significant (Table 2). Those who were married had three times increased odds of experiencing IPV compared to women with a steady partner [OR 3.05, 95\% CI: 1.00-9.30, $\mathrm{p}=0.0495]$. Participants with few symptoms of depression (low SRQ score) had an $80 \%$ decrease in odds of having any IPV as compared those with high SRQ score [95\% CI: $0.10-0.38, \mathrm{p}<0.0001]$. Multivariable logistic regression was attempted by including 4 variables (friend social support, education, marital status, depression) in the model selection, however, only SRQ score category was selected. Thus, no results from multivariable logistic regression are reported.

\section{HIV Outcomes at One-month follow-up and the Relationship to IPV}

At the one-month follow-up, $74 \%$ of women had disclosed to their partners ( $\mathrm{n}=195)$ and $84 \%$ had started ART $(\mathrm{n}=220)$ (Table 3). Only one participant had not started ART $(<1 \%)$, the remaining had missing data $(16 \%)$. Of those with adherence data measured by pill count, $82 \%$ had adherence $\geq 90 \%$ ( $n=163$ ). Self-reported non-adherence, measured by a missed dose in the past month, was higher amongst women reporting IPV compared to women not reporting IPV $(26 \%$ $\mathrm{v} 14 \%, \mathrm{p}=0.0499)$. Women who experience IPV were twice as likely to miss a dose in the past month (OR: 2.08 95\% CI: [1.11-3.90]). There was no significant relationship between IPV and any other outcome including ART initiation, partner disclosure, adherence self-efficacy, or retention in care at one month.

\section{Discussion}

We found that among PWLWH in Malawi in our study population, prevalence of IPV was high (39\%). Most women reporting IPV experienced more than one type of violence $(53 \%)$. IPV was significantly associated with depression and being married. For women retained at the one-month followup visit, IPV was also associated with reporting a missed dose in the past month but not with adherence measured by pill count. 
Prevalence of IPV in our study (39\%) was similar to the WHO African regional lifetime prevalence of violence against all women $(36 \%),{ }^{54}$ prevalence in SSA $(44 \%),{ }^{1}$ and to the lifetime estimates of IPV in Malawi (38\%). ${ }^{29}$ Measures of IPV, including tools and definitions used, make a difference in how IPV prevalence is interpreted. We used the WHO IPV tool and asked whether the woman had ever experienced IPV from her current partner, whereas other studies assessed IPV in the past year, lifetime prevalence from any partner, or specifically IPV during her current pregnancy. This could explain why our study found higher prevalence compared to similar studies conducted in SSA that ranged from $21 \%{ }^{13}$ to $32 \%{ }^{15}$ of PWLWH having experienced IPV in the past year. As with any self-reported measure, there is potential for social desirability bias which could have resulted in under-reporting of IPV. Additionally, the Malawian context may lead to underreporting due to participants considering IPV a personal issue that should remain private within a household. ${ }^{55}$ Although our study staff were all experienced with counseling and attempted to build rapport with the study participants to create a comfortable environment, it is possible that women did not fully report IPV for fear of repercussions from her partner, or fear of judgement; hence the true prevalence of IPV may be higher.

In this analysis, around half (53\%) of women reporting IPV experienced more than one type of violence, which was similar to findings in South Africa (48\%). ${ }^{13}$ In our study population emotional, sexual, and physical violence occurred at similar frequency; between $21-24 \%$ of women experiencing each IPV type. These findings are consistent with a study in Cameroonian HIV positive women which found $18-29 \%$ of HIV positive women (not just pregnant women) experienced each IPV type. ${ }^{56}$ Twenty-one percent of our participants experienced sexual violence, which is similar to findings from a study in Malawi amongst pregnant women (both those living with or without HIV) $(29 \%) \cdot{ }^{31}$ However, other studies in the region among PWLWH show varying results, with $2 \%$ in South Africa experiencing sexual violence in the past 12 months ${ }^{13}$ and $34 \%$ in Zambia experiencing sexual violence ever by their current partner (34\%). ${ }^{16}$ Regarding physical violence, $23 \%$ of the study population experienced this type of IPV ever in her current relationship. These findings are similar to other studies among PWLWH in South Africa $(20 \%),{ }^{57}$ and the lifetime prevalence of physical violence in all Malawian women $(20 \%){ }^{58}$

IPV was higher among married women compared to women with a steady partner. This is perhaps due to the fact that violence may be normalized in marital relationships in Malawi. ${ }^{55}$ There was a significant association between SRQ (depression) and IPV, which is unsurprising given prior research. Several other studies have also reported this relationship among the pregnant population regardless of HIV status. ${ }^{22,25,59}$ Since IPV was collected at one time point, we cannot comment on the direction of the association. Our analysis did not find an association between IPV and alcohol/drug abuse seen in other studies; ${ }^{60,61}$ this may be because the rates of drinking (4\%) or having a drug related problem $(<1 \%)$ were very low.

Self-reported non-adherence, measured by a missed dose in the past month, was higher in women reporting IPV. This is consistent with findings among non-pregnant HIV positive women ${ }^{18}$ and studies on PWLWH. ${ }^{19,20}$ It should be noted however, that HIV outcomes were measured at the one-month follow up visit, therefore were only assessed on participants retained and may not reflect the overall study population. To reduce missing data, participants who missed the follow-up visit were traced using locator information given at enrollment, but some could not be reached.

We found no difference in partner disclosure amongst women experiencing IPV as compared to those not reporting IPV, whereas some studies have reported that women who experience IPV were less likely to disclose. ${ }^{16,62}$ However, these studies also suggest a bidirectional association between status disclosure and IPV. Since we do not have follow up IPV assessments we do not know if there was an increase in IPV after disclosing, although this is an important area for future research. IPV was not associated with starting ART, although there was only one participant who did not start ART. There was also no association between IPV and adherence measured by pill count, or adherence self-efficacy even though there is an association in self-reported nonadherence (measured by missed doses). This may be because a pill count requires more missed doses $(>3)$ to influence adherence $(<90 \%)$. If a participant only missed one dose in the past month, her pill count would show good adherence $(>90 \%)$ but she would be counted as non-adherent by selfreported missed doses. Additionally, the participants who started ART were still in the first month of their lifelong treatment at the time follow-up data were collected, and it is possible IPV may have a delayed effect on outcomes.

The high prevalence of IPV among PWLWH in this study $(39 \%)$ demonstrates that additional attention on IPV is essential to identify mechanisms to prevent, screen, and manage IPV amongst PWLWH. Our study, which took place in antenatal clinic, adds to prior findings that screening and risk assessment in antenatal care may be an important starting point for identifying IPV among pregnant women. ${ }^{63-65}$ Upon identification of IPV experience or risk, a variety of services have been shown to be effective among pregnant women, including active referral to psychosocial support and counselling to reduce anxiety and depression, ${ }^{66}$ safety planning, linking to resources such as communitybased support. ${ }^{67}$ However, most of these solutions have been studied in high-income settings, ${ }^{68}$ and data on effective scalable clinical based interventions for settings like Malawi are lacking. One recent intervention model in South Africa ("Safe and Sound") provides a package of care in antenatal clinic, and findings from the health care workers expressed the importance of integrating HIV services with the IPV intervention. ${ }^{69}$ Integration of service delivery for IPV prevention and intervention in antenatal and ART care may facilitate access to services that support PWLWH in Malawi.

\section{Conclusion}

To our knowledge, this analysis was the first to measure the prevalence of IPV, identify sociodemographic characteristics associated with IPV, as well as assess potential associations with early outcomes amongst pregnant women living with HIV not yet on ART in Malawi. In this study we asked IPV only at the time of enrollment, and therefore cannot assume directionality of associations. Further studies are needed to examine how IPV changes over time in PWLWH in Malawi and factors associated with increased risk of IPV, such as HIV status disclosure. This study corroborates existing literature that IPV is a global issue that needs focus in sub-Saharan Africa and demonstrates this problem among PWLWH in Malawi. IPV screening as well as interventions 
targeted to this subgroup are important to support PWLWH and optimize HIV outcomes.

\section{Acknowledgements}

We are thankful to the Malawi Ministry of Health for support and partnership. We are grateful to the pregnant women living with HIV who participated in this study. We acknowledge the support of the VITAL Start research team for their hard work and dedication.

\section{Author contributions}

$\mathrm{ECW}$ wrote the manuscript with support from MHK. MHK designed the VITAL Start study and obtained the grant, as well as ethical approval. SA, T'T, AM, MJC, and EJA provided input on study design. TT and AM oversaw the study and provided management to the study team. XY provided data management and analysis. All authors contributed to the manuscript review and final approval prior to submission.

\section{Disclosure statement}

The authors report no conflicts of interest.

\section{Ethics and Consent}

The VITAL Start study has been approved by the Malawi National Health Science Research Committee (protocol\#16/05/1593) and the Baylor College of Medicine Institutional Review Board. All participants provided written, informed consent.

Funding information

The National Institute of Mental Health (NIMH) funded the VITAL Start study (R01MH115793). The funders played no role in the design, analysis, or any preparation of the manuscript.

\section{Paper context}

Intimate partner violence can have detrimental health and psychosocial effects on pregnant women living with HIV (PWLWH). To our knowledge, this study was the first to quantify IPV among PWLWH in the Malawian setting. We identify sociodemographic characteristics associated with IPV and assess potential associations with early outcomes amongst PWLWH in Malawi. We conclude that IPV is a problem facing PWLWH in Malawi, and specific interventions may be needed to support this population.

\section{References}

1.Muluneh MD, Stulz V, Francis L, Agho K. Gender based violence against women in Sub-Saharan africa: a systematic review and metaanalysis of cross-sectional studies. Int $\mathrm{J}$ Environ Res Public Health. 2020;17(3):903. DOI: 10.3390/ijerph17030903

2.CDC. Intimate Partner Violence 2018 [Internet]. Cited 2020 June 5. Available from: https://www.cdc.gov/violenceprevention/ intimatepartnerviolence/index.html.

3.Shah PS, Shah J. Maternal exposure to domestic violence and pregnancy and birth outcomes: a systematic review and meta-analyses J Womens Health. 2010;19(11):2017-31. DOI: 10.1089/jwh.2010.2051

4.Alhusen JL, Ray E, Sharps P, Bullock L. Intimate partner violence during pregnancy: maternal and neonatal outcomes. J Womens Health. 2015;24(1):100-6. DOI: 10.1089/jwh.2014.4872

5.Murphy CC, Schei B, Myhr TL, Du Mont J. Abuse: a risk factor for low birth weight? A systematic review and meta-analysis. CMAJ. 2001;164(11):1567-72.

6.Koen N, Wyatt GE, Williams JK, et al. Intimate partner violence: associations with low infant birthweight in a South African birth cohort. Metab Brain Dis. 2014;29(2):281-99. DOI: 10.1007/s11011-014-95254

7.Donovan B, Spracklen C, Schweizer M, Ryckman K, Saftlas A. Intimate partner violence during pregnancy and the risk for adverse infant outcomes: a systematic review and meta-analysis. Int J Gynecol Obstet. 2016;123(8):1289-99. DOI: 10.1111/1471-0528.13928

8.Rao N, Norris Turner A, Harrington B, Nampandeni P, Banda V, Norris A. Correlations between intimate partner violence and spontaneous abortion, stillbirth, and neonatal death in rural Malawi. Int J Gynecol Obstet. 2017;138(1):74-8. DOI: 10.1002/ijgo.12173

9.Berhanie E, Gebregziabher D, Berihu H, Gerezgiher A, Kidane G. Intimate partner violence during pregnancy and adverse birth outcomes: a case-control study. Reprod Health. 2019;16(1):22. DOI: 10.1186/ s12978-019-0670-4

10.Memiah P, Bond T, Opanga Y, et al. Neonatal, infant, and child mortality among women exposed to intimate partner violence in East Africa: a multi-country analysis. BMC Women's Health. 2020;20(1):10. DOI: $10.1186 / \mathrm{s} 12905-019-0867-2$

11.Ntaganira J, Muula AS, Masaisa F, Dusabeyezu F, Siziya S, Rudatsikira E. Intimate partner violence among pregnant women in Rwanda. BMC Women's Health. 2008;8(1):17. DOI: 10.1186/14726874-8-17

12.Shamu S, Abrahams N, Temmerman M, Musekiwa A, Zarowsky C. A systematic review of African studies on intimate partner violence against pregnant women: prevalence and risk factors. PloS One. 2011;6(3):e17591. DOI: 10.1371/journal.pone.0017591

13.Bernstein M, Phillips T, Zerbe A, et al. Intimate partner violence experienced by HIV-infected pregnant women in South Africa: a cross-sectional study. BMJ Open. 2016;6(8):e011999. DOI: 10.1136/ bmjopen-2016-011999

14.Ezeanochie MC, Olagbuji BN, Ande AB, Kubeyinje WE, Okonofua FE. Prevalence and correlates of intimate partner violence against HIV-seropositive pregnant women in a Nigerian population. Acta Obstet Gynecol Scand. 2011;90(5):535-9. DOI: 10.1111/j.16000412.2011.01083.x

15.Gichane MW, Moracco KE, Thirumurthy H, Okitolonda EW, Behets $\mathrm{F}$, Yotebieng $\mathrm{M}$. Intimate partner violence and prevention of mother to child transmission of HIV: Evidence from Kinshasa, Democratic Republic of Congo. PloS One. 2018;13(8):e0203471. DOI: 10.1371/ journal.pone. 0203471

16.Hampanda KM, Rael CT. HIV status disclosure among postpartum women in Zambia with varied intimate partner violence experiences. AIDS Behav. 2018;22(5):1652-61. DOI: 10.1007/s10461-017-1909-0

17.Groves AK, Reyes HLM, Moodley D, Maman S. HIV Positive diagnosis during pregnancy increases risk of IPV postpartum among women with no history of IPV in their relationship. AIDS Behav. 2018;22(6):1750-7. DOI: 10.1007/s10461-017-1868-5

18.Hatcher AM, Smout EM, Turan JM, Christofides N, Stoeck1 H. Intimate partner violence and engagement in HIV care and treatment among women: a systematic review and meta-analysis. AIDS. 2015;29(16):2183-94. DOI: 10.1097/QAD.0000000000000842

19. Yee LM, Janik MC, Dorman RM, Chong PS, Garcia PM, Miller ES. Relationship between intimate partner violence and antiretroviral adherence and viral suppression in pregnancy. Sex Reprod Healthc. 2018;17:7-11.

20.Hampanda KM. Intimate partner violence and HIV-positive women's non-adherence to antiretroviral medication for the purpose of prevention of mother-to-child transmission in Lusaka, Zambia. Soc Sci Med. 2016;153:123-30. DOI: 10.1016/j.socscimed.2016.02.011

21.Kim MH, Zhou A, Mazenga A, et al. Why did I stop? Barriers and facilitators to uptake and adherence to ART in Option B+ HIV care in Lilongwe, Malawi. PloS One. 2016;11(2):e0149527. DOI: 10.1371/ 
journal.pone.0149527

22.Tsai AC, Tomlinson M, Comulada WS, Rotheram-Borus MJ. Intimate partner violence and depression symptom severity among South African women during pregnancy and postpartum: populationbased prospective cohort study. PLoS Med. 2016;13(1):e1001943. DOI: 10.1371/journal.pmed.1001943

23.Stewart RC, Umar E, Tomenson B, Creed F. A cross-sectional study of antenatal depression and associated factors in Malawi. Arch Womens Ment Health. 2014;17(2):145-54. DOI: 10.1007/s00737-013-0387-2.

24.Halim N, Beard J, Mesic A, Patel A, Henderson D, Hibberd P. Intimate partner violence during pregnancy and perinatal mental disorders in low and lower middle income countries: A systematic review of literature, 1990-2017. Clin Psychol Rev. 2018;66:117-35. DOI: $10.1016 /$ j.cpr.2017.11.004

25.Rogathi JJ, Manongi R, Mushi D, et al. Postpartum depression among women who have experienced intimate partner violence: A prospective cohort study at Moshi, Tanzania. J Affect Disord. 2017;218:238-45. DOI: $10.1016 /$ j.jad.2017.04.063

26.Gonzalez JS, Batchelder AW, Psaros C, Safren SA. Depression and HIV/AIDS treatment nonadherence: a review and metaanalysis. J Acquir Immune Defic Syndr. 2011;58(2). DOI: 10.1097/ QAI.0b013e31822d490a

27.Hartzell JD, Janke IE, Weintrob AC. Impact of depression on HIV outcomes in the HAART era. J Antimicrob Chemother. 2008;62(2):24655. DOI: $10.1093 / \mathrm{jac} / \mathrm{dkn} 193$

28.Onono M, Odwar T, Abuogi L, et al. Effects of Depression, Stigma and Intimate Partner Violence on Postpartum Women's Adherence and Engagement in HIV Care in Kenya. AIDS Behav. 2019:1-9. DOI: 10.1007/s10461-019-02750-y

29.Global Database on Violence against Women [Internet]. 2016. Available from: http://evaw-global-database.unwomen.org/en/ countries/africa/malawi.

30.Malawi National Statistical Office (NSO) and ICF. 2017. Malawi Demographic and Health Survey 2015-16. Zomba, Malawi, and Rockville, Maryland, USA.

31.Chasweka R, Chimwaza A, Maluwa A. Isn’t pregnancy supposed to be a joyful time? A cross-sectional study on the types of domestic violence women experience during pregnancy in Malawi. Malawi Med J. 2018;30(3):191-6. DOI: 10.4314/mmj.v30i3.11

32.Kim MH, Ahmed S, Tembo T, et al. VITAL Start: Video-Based Intervention to Inspire Treatment Adherence for Life-Pilot of a Novel Video-Based Approach to HIV Counseling for Pregnant Women Living with HIV. AIDS Behav. 2019:1-12. DOI: 10.1007/s10461-019-02634-1

33.Ministry of Health. Malawi Population-based HIV Impact Assessment (MPHIA) 2015-16: first report. 2017.

34.Wolrd Health Organization (WHO). WHO Violence Against Women Instrument. 2003.

35.Ellsberg MC, Heise L. Researching violence against women: a practical guide for researchers and activists. 2005. World Health Organization. 257 p.

36.Beusenberg M, Orley JH, World Health Organization. A User's guide to the self reporting questionnaire (SRQ). Geneva: World Health Organization; 1994.

37.Stewart RC, Kauye F, Umar E, et al. Validation of a Chichewa version of the self-reporting questionnaire (SRQ) as a brief screening measure for maternal depressive disorder in Malawi, Africa. J Affect Disord. 2009;112(1-3):126-34. DOI: 10.1016/j.jad.2008.04.001

38.Zimet GD, Dahlem NW, Zimet SG, Farley GK. The multidimensional scale of perceived social support. J Pers Assess. 1988;52(1):30-41. DOI: 10.1207/s15327752jpa5201_2

39.Stewart RC, Umar E, Tomenson B, Creed F. Validation of the multi-dimensional scale of perceived social support (MSPSS) and the relationship between social support, intimate partner violence and antenatal depression in Malawi. BMC Psychiatry. 2014;14(1):180. DOI: $10.1186 / 1471-244 X-14-180$

40.Saunders JB, Aasland OG, Babor TF, De la Fuente JR, Grant M. Development of the alcohol use disorders identification test (AUDIT): WHO collaborative project on early detection of persons with harmful alcohol consumption-II. Addiction. 1993;88(6):791-804. DOI: 10.1111/ j.1360-0443.1993.tb02093.x

41.Bradley KA, Bush KR, Epler AJ, et al. Two brief alcohol-screening tests From the Alcohol Use Disorders Identification Test (AUDIT): validation in a female Veterans Affairs patient population. Arch Intern Med. 2003;163(7):821-9. DOI: 10.1001/archinte.163.7.821

42.Zverev Y. Problem drinking among university students in Malawi. Coll Antropol. 2008;32(1):27-31.

43.Berman AH, Bergman H, Palmstierna T, Schlyter F. DUDIT. The Drug Use Disorders Identification Test-E MANUAL. Karolinska Institutet, Stockholm. 2003.

44.Voluse AC, Gioia CJ, Sobell LC, Dum M, Sobell MB, Simco ER. Psychometric properties of the Drug Use Disorders Identification Test (DUDIT) with substance abusers in outpatient and residential treatment. Addict Behav. 2012;37(1):36-41. DOI: 10.1016/j.addbeh.2011.07.030.

45.Lancaster KE, Go VF, Lungu T, et al. Substance use and HIV infection awareness among HIV-infected female sex workers in Lilongwe, Malawi. Int J Drug Policy. 2016;30:124-31. DOI: 10.1016/j. drugpo.2016.02.020

46.Babor TF, de la Fuente JR, Saunders J, Grant M. The Alcohol Use Disorders Identification Test: Guidelines for use in primary health care. 2001.

47.Chesney MA, Ickovics J, Chambers D, et al. Self-reported adherence to antiretroviral medications among participants in HIV clinical trials: the AACTG adherence instruments. AIDS Care. 2000;12(3):255-66. DOI: $10.1080 / 09540120050042891$

48.Johnson MO, Neilands TB, Dilworth SE, Morin SF, Remien RH, Chesney MA. The role of self-efficacy in HIV treatment adherence: validation of the HIV Treatment Adherence Self-Efficacy Scale (HIVASES). J Behav Med. 2007;30(5):359-70. DOI: 10.1007/s10865-0079118-3

49.Rosenberg NE, Mtande TK, Saidi F, et al. Recruiting male partners for couple HIV testing and counselling in Malawi's option B+ programme: an unblinded randomised controlled trial. Lancet HIV. 2015;2(11):e483-e91. DOI: 10.1016/S2352-3018(15)00182-4

50.Phillips T, Brittain K, Mellins CA, et al. A self-reported adherence measure to screen for elevated HIV viral load in pregnant and postpartum women on antiretroviral therapy. AIDS Behav. 2017;21(2):450-61. DOI: $10.1007 / \mathrm{s} 10461-016-1448-0$

51.Wilson IB, Fowler FJ, Cosenza CA, et al. Cognitive and field testing of a new set of medication adherence self-report items for HIV care. AIDS Behav. 2014;18(12):2349-58. DOI: 10.1007/s10461-013-0610-1

52.Hess LM, Raebel MA, Conner DA, Malone DC. Measurement of adherence in pharmacy administrative databases: a proposal for standard definitions and preferred measures. Ann Pharmacother. 2006;40(7-8):1280-8. DOI: 10.1345/aph.1H018

53. Ortego C, Huedo-Medina TB, Llorca J, et al. Adherence to highly active antiretroviral therapy (HAART): a meta-analysis. AIDS Behav. 2011;15(7):1381-96. DOI: 10.1007/s10461-011-9942-x

54.World Health Organization (WHO), London School of Hygiene and Tropical Medicine, South African Medical Research Council. Global and regional estimates of violence against women: Prevalence and health effects of intimate partner violence and non-partner sexual violence. 2013. $51 \mathrm{p}$.

55.Chepuka L, Taegtmeyer M, Chorwe-Sungani G, Mambulasa J, Chirwa E, Tolhurst R. Perceptions of the mental health impact of intimate partner violence and health service responses in Malawi. Glob 
Health Action. 2014;7(1):24816. DOI: 10.3402/gha.v7.24816

56.Fiorentino M, Sagaon-Teyssier L, Ndiaye K, et al. Intimate partner violence against HIV-positive Cameroonian women: Prevalence, associated factors and relationship with antiretroviral therapy discontinuity-results from the ANRS-12288 EVOLCam survey. Women's Health. 2019. DOI: 10.1177/1745506519848546

57.Matseke G, Rodriguez VJ, Peltzer K, Jones D. Intimate partner violence among HIV positive pregnant women in South Africa. J Psychol Afr. 2016;26(3):259-66.

58.Bazargan-Hejazi S, Medeiros S, Mohammadi R, Lin J, Dalal K. Patterns of intimate partner violence: a study of female victims in Malawi. J Inj Violence Res. 2013;5(1):38. DOI: 10.5249/jivr.v5i1.139

59.Nduna M, Jewkes RK, Dunkle KL, Shai NPJ, Colman I. Associations between depressive symptoms, sexual behaviour and relationship characteristics: a prospective cohort study of young women and men in the Eastern Cape, South Africa. J Int AIDS Soc. 2010;13(1):44. DOI: $10.1186 / 1758-2652-13-44$

60.Foran HM, O'Leary KD. Alcohol and intimate partner violence: A meta-analytic review. Clin Psychol Rev. 2008;28(7):1222-34. DOI: 10.1016/j.cpr.2008.05.001

61.Tenkorang EY, Asamoah-Boaheng M, Owusu AY. Intimate partner violence (IPV) against HIV-positive women in Sub-Saharan Africa: a mixed-method systematic review and meta-analysis. Trauma Violence Abuse. 2020. DOI: 10.1177/1524838020906560

62.Hatcher AM, Woollett N, Pallitto CC, et al. Bidirectional links between HIV and intimate partner violence in pregnancy: implications for prevention of mother-to-child transmission. J Int AIDS Soc. 2014;17(1):19233. DOI: 10.7448/IAS.17.1.19233
63.Chisholm CA, Bullock L, Ferguson II JEJ. Intimate partner violence and pregnancy: screening and intervention. Am J Obstet Gynecol. 2017;217(2):145-9. DOI: 10.1016/j.ajog.2017.05.043

64.Bianchi AL, Cesario SK, McFarlane J. Interrupting intimate partner violence during pregnancy with an effective screening and assessment program. J Obstet Gynecol Neonatal Nurs. 2016;45(4):579-91. DOI: 10.1016/j.jogn.2016.02.012

65.Daley D, McCauley M, van den Broek N. Interventions for women who report domestic violence during and after pregnancy in lowand middle-income countries: a systematic literature review. BMC Pregnancy Childbirth. 2020;20(1):1-9. DOI: 10.1186/s12884-0202819-0

66.Hameed M, O’Doherty L, Gilchrist G, Tirado-Muñoz J, Taft A, Chondros $\mathrm{P}$, et al. Psychological therapies for women who experience intimate partner violence. Cochrane Database Syst Rev. 2020(7). DOI: 10.1002/14651858.CD013017.pub2

67.Turan JM, Hatcher AM, Odero M, Onono M, Kodero J, Romito $\mathrm{P}$, et al. A community-supported clinic-based program for prevention of violence against pregnant women in rural Kenya. AIDS Res Treat. 2013. DOI: $10.1155 / 2013 / 736926$

68. Young CR, Arnos DM, Matthews LT. A scoping review of interventions to address intimate partner violence in sub-Saharan African healthcare. Glob Public Health. 2019;14(9):1335-46.

69.Sprague C, Woollett N, Hatcher AM. Enhancing agency for health providers and pregnant women experiencing intimate partner violence in South Africa. Glob Public Health. 2020;15(12):1820-35. 